\title{
Maternal Depression, Adverse Childhood Experiences, and Social Support in Relation to Gestational Diabetes Risk
}

Margaret E. Versteegen ( $\sim$ meggwin@gmail.com )

University of Albany School of Public Health https://orcid.org/0000-0003-1848-8772

Christine T. Bozlak

University at Albany Health Sciences Campus: University at Albany Health Sciences Center

Heather Larkin

University at Albany School of Social Welfare

Allison A. Appleton

University at Albany Health Sciences Campus: University at Albany Health Sciences Center

\section{Research article}

Keywords: gestational diabetes, psychosocial factors, depression, adverse childhood experiences, social support

Posted Date: February 12th, 2021

DOI: https://doi.org/10.21203/rs.3.rs-228057/v1

License: () (1) This work is licensed under a Creative Commons Attribution 4.0 International License. Read Full License 


\section{Abstract \\ Purpose}

Psychosocial factors are of increasing interest as potential influencers in disease development. This study explores associations between gestational diabetes mellitus (GDM) and maternal depression, adverse childhood experiences (ACEs), and social support, in response to emerging evidence in these areas.

\section{Methods}

An observational, prospective cohort study (AIMS) served as the source of secondary data for this study. Participants included 300 pregnant women aged $18-$ 40 years at an upstate New York prenatal care clinic, who completed a set of self-report questionnaires assessing exposures and stressors both during and prior to their pregnancy. Data were also abstracted from infant and maternal medical records.

\section{Results}

Logistic regression modeling estimated the odds ratios (ORs) of developing GDM in relation to psychosocial factors. There was a significant association between depression and GDM $(\mathrm{OR}=2.85,95 \% \mathrm{Cl}: 1.15,7.06)$, which persisted in the model adjusted for age and $\mathrm{BMI}(\mathrm{aOR}=3.19,95 \% \mathrm{Cl}: 1.25,8.10)$. $\mathrm{No}$ significant associations were found between ACEs or social support with GDM.

\section{Conclusions}

Study findings support an association between maternal depression and GDM development. This study underscores the need for additional research on psychosocial factors and connections to health risks.

\section{Introduction}

Gestational diabetes mellitus (GDM) is a form of diabetes, or abnormal blood glucose (sugar) metabolism, diagnosed during pregnancy. Pregnant, nondiabetic women who are initially noted to experience high blood glucose levels during their pregnancy are considered to have GDM (ADA, 2016). The causes of GDM are complex and not fully understood, yet it is becoming a common pregnancy complication with significant implications for infant and mother morbidity and mortality, in addition to developmental concerns later in life.

GDM prevalence rates have increased nationwide, affecting 9.2\% of all United States' (US) pregnancies (DeSisto et al., 2014). Some common risk factors have been identified, primarily with regards to maternal demographics and clinical characteristics, like higher age and body mass indices (BMls). However, a review of the literature reveals some discrepancies and variations in these risk factors, including a number of underexplored psychosocial risk factors.

The biopsychosocial model (Engel, 1977) acknowledges the importance of taking a more holistic view of individuals and health outcomes, and prominently takes into account factors that affect health from not just the biological sphere, but equally from the social and psychological domains. Limited research has been conducted on psychosocial factors, behaviors, and early life exposures and their possible latent effects on pregnancy. This provides an important area of focus for intervention measures, and a better understanding of risk relationships is necessary.

Psychosocial factors, like depression, adverse childhood experiences (ACEs), and social support, have been linked to a wide range of health outcomes. Maternal depression is a risk factor for several different negative pregnancy outcomes and has been linked to GDM in recent research (Bowers et al., 2013; Byrn \& Penckofer, 2015; Hinkle et al., 2016; Horsch et al., 2016). Depression has also been shown to have a negative association with health behaviors that might mitigate GDM risk in pregnant women, including healthy dietary intakes, exercise, and stress-management (Omidvar et al., 2018).

ACEs refer to stressful or traumatic events that occur before age 18, and ramifications of the cumulative stress from ACEs have been found to increase adult risk for several chronic diseases including coronary heart disease, stroke, obesity, and depression (CDC, 2019). Negative experiences during critical childhood and adolescent developmental periods can create a life-long burden that may be particularly evident during pregnancy. Mason et al. (2016) appear to have been the first to test for associations between GDM and two of the four ACE domains, physical and/or sexual abuse, finding a dose-response relationship with GDM risk that did not attenuate with adjustment for overweight/obesity.

Low well-being (Sattler et al., 2017) and unhealthy nutritional intakes (Omidvar et al., 2018) have been linked with low levels of social support in pregnant women, and these can have a negative impact on pregnancy concerns like GDM. While pregnancy is a natural physiological process, it can be an overwhelming experience for some women, and is replete with physical and emotional changes. Pregnancy adaptation has been found to be enhanced in women with higher levels of social support, and these women reported significantly less stress compared to women with lower levels of social support (Chou et al., 2008). Emerging evidence suggests connections between maternal social support and GDM risk. Mizuno et al. (2016) found a woman's level of emotional support and neighborhood trust was significantly and independently associated with GDM prevalence during the second or third trimester; this illuminates how poor emotional support can be stressful and cause hormone release, leading to increased insulin resistance in the body.

Research on connections between depression and GDM is still evolving, and limited with regards to associations with ACEs and social support. The objectives of this study were to further explore associations between depression and GDM, and address literature gaps considering ACEs and social support as contributors to GDM risk. 


\section{Materials And Methods}

Data were derived from the Albany Infant and Mother Study (AIMS), an observational, prospective cohort study. Between June 2015 and February 2018,300 pregnant women who received prenatal care at an obstetrics clinic in upstate New York were enrolled on average at 27 weeks gestation. Details of this study can be found elsewhere (Appleton et al., 2019). Inclusion criteria were singleton pregnancy, maternal age between 18 and 40 years, English-speaking, and planned to give birth at the clinic's medical center. All participants provided documented informed consent and completed a self-report questionnaire packet. After birth, a structured abstraction of clinical information regarding maternal health, delivery, and infant characteristics was conducted by physicians. Of the 300 who enrolled, 266 had available information on all psychosocial study variables and were included in analysis. Twenty-two women (8.3\%) had a diagnosis of GDM. The study protocol was approved by the [Name Redacted for Review] Institutional Review Board.

The present analysis considers three different psychosocial predictor variables measured by validated instruments and includes: the Edinburgh Postnatal Depression Scale (EPDS; Cox et al., 1987), the ACES questionnaire (Felitti \& Anda, 2010), and the Interpersonal Support Evaluation List (ISEL; Cohen \& Hoberman, 1983).

The EPDS is comprised of ten questions that assess depressive symptoms in the past week, each scored on a four-point scale (0-3) for possible scores ranging from 0-30 (Cox et al., 1996). The EPDS is widely used and has been validated in women during pregnancy ( $a=0.82$; Bergink et al., 2011), with higher scores indicative of more depressive symptoms. Participant scores were considered on a continuum and as categorical predictors. Given that participants completed the EPDS primarily during their second trimester, depression was dichotomized as 'yes' and 'no' with presence of depression defined as a score > 10 (Bergink et al., 2011).

The ACES questionnaire is a 10-item self-report tool developed after the original ACE study by Felliti et al. (1998; Felitti \& Anda, 2010). It provides a retrospective identification of childhood trauma and stressful experiences that occurred prior to age 18, with total points scored out of ten. Given the extensive literature that has shown a variety of negative health outcomes with a graded, dose-response relationship with ACEs (CDC, 2019), participant ACE scores were used as a continuous measure. ACE scores were also dichotomized into high ( $\geq 4)$ and low $(<4)$, as a significantly increased risk for negative health outcomes has been identified in those with an ACE score $\geq 4$ (Felitti et al., 1998). Analytical power was too low to provide stable estimates with the 5-category and abuse category ACE variables (data not shown).

The ISEL (Cohen \& Hoberman, 1983) is a widely used measure of general social support developed in response to the identified moderating effect of certain factors on stressful and/or negative life events, and the desire to better quantify the positive role of social support. Higher ISEL-12 survey scores indicate greater perceived social support. ISEL-12 scores typically range from 0-36; in the AIMS dataset, derived scores ranged from 12-48 and were used for analysis in this current study for ease and continuity. ISEL scores were examined continuously and categorically. No 'cut-off' values have been established for the ISEL12 , so tertiles of the sample scores were used to establish categorical groups with 'low' (bottom two tertiles) and 'high' (top tertile) social support.

The main outcome of GDM was diagnosed in study participants using a standard oral glucose tolerance test (OGTT); this was performed as part of routine prenatal care at the obstetrics clinic between 24-27 weeks, and results (yes/no) were extracted from medical records. Based on the literature, multiple covariates were considered as potential factors involved in GDM risk. Age and gestational weight gain were extracted from medical records. Participant BMI was calculated from the height and pre-pregnancy weight information documented in the medical records. Race, ethnicity, income level, education level, and marital status were obtained from the demographic survey portion of the study packet.

Preliminary bivariate analyses (data not shown) indicated a significantly higher risk for GDM in women aged $\geq 30$, and in women with obesity (pre-pregnancy $\mathrm{BMI} \geq 30$ ); these covariates were thus used in regression models described below. No significant associations were seen in bivariate analyses for race, ethnicity, education, income, marital status, categorical pregnancy weight gain, or when categorizing age or BMI in additional ways, and thus were not added to the regression models testing the associations between psychosocial factors and GDM.

To test the hypothesis that maternal depression, ACEs, and social support are associated with GDM, sets of logistic regression models were fit for each construct with the binary GDM variable as the outcome. Odds ratios (ORs) and 95\% confidence intervals (95\% Cls) were estimated. For each psychosocial factor, unadjusted and models adjusted for covariates were fit. An unadjusted model was first created for the different predictor variables (Model 1 ), followed by a minimally controlled model using the age $\geq 30$ covariate (Model 2 ), and finally a third model that controlled for both age $\geq 30$ and BMI $\geq 30$ (Model 3 ). All analyses were conducted using IBM SPSS Statistics, Version 25. A $p$ value $\leq 0.05$ was considered significant.

\section{Results}

On average, participants were 28.5 years old, with a pre-pregnancy BMI of 29.02 (overweight category as per CDC guidelines). Examining BMI categorically, the two highest frequency categories were obese women (37.6\%) and normal weight women (35.2\%). Participants gained 12.01 kilograms (kg; or 26.46 pounds) on average during their pregnancy. Looking at pregnancy weight gain categorically, in terms of recommended weight gain based on pre-pregnancy BMI, the highest frequency category was those who gained more than the recommended amount of weight with almost half of all participants (47.5\%). Just over half of study participants classified themselves as White, Non-Hispanic (52.4\%), and 11.9\% identified as being Latina/Hispanic. Many women were low-income; the most common annual income category reported by $19 \%$ of participants was $<\$ 9000$. The majority of women had achieved a $>$ high school education level (62.0\%), and were not married (62.4\%). Participant characteristics are presented in Table 1. 
Table 1

Participant Demographics

\begin{tabular}{|c|c|c|}
\hline Continuous Variables & Mean & Standard Deviation \\
\hline Age & 28.54 & 5.48 \\
\hline Pre-pregnancy BMI & 29.02 & 8.78 \\
\hline Pregnancy weight gain $(\mathrm{kg})$ & 12.01 & 7.26 \\
\hline Categorical Variables & Frequency & Percent of total \\
\hline Race & 75 & 25.5 \\
\hline Black/AA, NH & 154 & 52.4 \\
\hline White, NH & 65 & 22.1 \\
\hline \multicolumn{3}{|l|}{ Other } \\
\hline Ethnicity & 35 & 11.9 \\
\hline Latino/Hispanic & 259 & 88.1 \\
\hline \multicolumn{3}{|l|}{ Not Latino/Hispanic } \\
\hline Income level & 56 & 19.0 \\
\hline$<\$ 9000$ & 38 & 12.9 \\
\hline$\$ 9000-19999$ & 47 & 16.0 \\
\hline$\$ 20000-29999$ & 19 & 6.5 \\
\hline$\$ 30000-39999$ & 19 & 6.5 \\
\hline$\$ 40000-49999$ & 22 & 7.5 \\
\hline$\$ 50000-79999$ & 12 & 4.1 \\
\hline$\$ 80000-99999$ & 39 & 13.3 \\
\hline$>\$ 100000+$ & 30 & 10.2 \\
\hline Don't know & 12 & 4.1 \\
\hline \multicolumn{3}{|l|}{ Prefer not to answer } \\
\hline Education level & 112 & 38.0 \\
\hline$\leq$ High School/GED & 183 & 62.0 \\
\hline \multicolumn{3}{|l|}{$>$ High School } \\
\hline Marital status & 111 & 37.6 \\
\hline Married & 184 & 62.4 \\
\hline \multicolumn{3}{|l|}{ Not married } \\
\hline Pregnancy weight gain $(\mathrm{kg})$ & 72 & 27.8 \\
\hline Gained recm'd wt & 64 & 24.7 \\
\hline Gained $<$ recm'd wt & 123 & 47.5 \\
\hline Gained > recm'd wt & & \\
\hline
\end{tabular}

The average EPDS score among participants was 8.70 (standard deviation (SD) 5.56). Examining binary EPDS scores, women with depression were found to be at significantly increased risk for GDM across all three models (Model 1, OR=2.85, 95\% Cl: 1.15, 7.06, p = 0.023; Model 2, aOR = 3.10, 95\% Cl: 1.23, 7.77, $p=$ 0.016; Model 3, aOR $=3.19,95 \% \mathrm{Cl}: 1.25,8.10, p=0.015)$. The strongest association was found in Model 3, with an almost 3.2 elevated odds of GDM after adjusting for both age and pre-pregnancy BMI. No significant associations were observed when depression was treated continuously (Table 2). 
Table 2

Logistic Regression Models for the Association between Depression and GDM

\begin{tabular}{|c|c|c|c|c|c|c|}
\hline & \multicolumn{2}{|l|}{ Model 1} & \multicolumn{2}{|l|}{ Model 2} & \multicolumn{2}{|l|}{ Model 3} \\
\hline & OR $(95 \% \mathrm{Cl})$ & $\mathrm{R}^{2}$ & aOR $(95 \% \mathrm{Cl})$ & $\mathrm{R}^{2}$ & aOR $(95 \% \mathrm{Cl})$ & $\mathrm{R}^{2}$ \\
\hline EPDS total & $1.05(0.98,1.14)$ & $1.4 \%$ & $1.05(0.98,1.13)$ & $4.7 \%$ & $1.04(0.97,1.13)$ & $8.5 \%$ \\
\hline EPDS binary & $2.85(1.15,7.06)^{\star}$ & $4.6 \%$ & $3.10(1.23,7.77)^{\star}$ & $8.5 \%$ & $3.19(1.25,8.10) \star$ & $12.7 \%$ \\
\hline \multicolumn{7}{|c|}{ *: denotes finding is statistically significant, $p \leq 0.05$} \\
\hline \multicolumn{7}{|c|}{ Model 1: unadjusted } \\
\hline \multicolumn{7}{|c|}{ Model 2: adjusted for age \pm 30} \\
\hline \multicolumn{7}{|c|}{ Model 3: adjusted for age $\pm 30, \mathrm{BMI} \pm 30$} \\
\hline Abbreviations & $O R=$ odds ratio; $a O$ & $=\operatorname{adjus}$ & ed odds ratio; $R^{2}=$ & gelke & $R^{2}$ & \\
\hline
\end{tabular}

The average ACE score among participants was 2.72 (SD 2.576). No significant associations were found in regression analyses for the various considerations of the ACE score variable and GDM (Table 3). Some trends towards increased GDM risk with the binary ACE variable were noted, although results were firmly nonsignificant.

Table 3

Logistic Regression Models for the Association between ACEs and GDM

\begin{tabular}{|c|c|c|c|c|c|c|}
\hline & \multicolumn{2}{|l|}{ Model 1} & \multicolumn{2}{|l|}{ Model 2} & \multicolumn{2}{|l|}{ Model 3} \\
\hline & OR $(95 \% \mathrm{Cl})$ & $\mathbf{R}^{2}$ & aOR $(95 \% \mathrm{Cl})$ & $\mathbf{R}^{2}$ & aOR $(95 \% \mathrm{Cl})$ & $\mathbf{R}^{2}$ \\
\hline ACEs total & $1.06(0.90,1.26)$ & $0.4 \%$ & $1.04(0.88,1.23)$ & $4.2 \%$ & $1.00(0.84,1.18)$ & $9.3 \%$ \\
\hline ACEs binary & $1.83(0.74,4.55)$ & $1.5 \%$ & $1.61(0.64,4.05)$ & $5.0 \%$ & $1.31(0.50,3.39)$ & $9.5 \%$ \\
\hline \multicolumn{7}{|c|}{ *: denotes finding is statistically significant, $p \leq 0.05$} \\
\hline \multicolumn{7}{|c|}{ Model 1: unadjusted } \\
\hline \multicolumn{7}{|c|}{ Model 2: adjusted for age \pm 30} \\
\hline \multicolumn{7}{|c|}{ Model 3: adjusted for age $\pm 30, \mathrm{BMI} \pm 30$} \\
\hline
\end{tabular}

The average ISEL score among AIMS participants was 38.63 (SD 7.33). No significant associations were observed for social support (either measured continuously or dichotomously) and GDM (Table 4), although a slight trend suggesting a protective effect of high levels of social support during pregnancy for GDM development was noted.

Table 4

Logistic Regression Models for the Association between Social Support and GDM

\begin{tabular}{|c|c|c|c|c|c|c|}
\hline & \multicolumn{2}{|l|}{ Model 1} & \multicolumn{2}{|l|}{ Model 2} & \multicolumn{2}{|l|}{ Model 3} \\
\hline & OR $(95 \% \mathrm{Cl})$ & $\mathrm{R}^{2}$ & aOR $(95 \% \mathrm{Cl})$ & $\mathrm{R}^{2}$ & aOR $(95 \% \mathrm{Cl})$ & $\mathbf{R}^{2}$ \\
\hline ISEL total & $0.98(0.93,1.04)$ & $0.3 \%$ & $0.98(0.93,1.04)$ & $3.7 \%$ & $0.99(0.93,1.05)$ & $7.7 \%$ \\
\hline ISEL binary & $0.61(0.23,1.61)$ & $0.9 \%$ & $0.60(0.22,1.59)$ & $4.4 \%$ & $0.64(0.24,1.73)$ & $8.3 \%$ \\
\hline \multicolumn{7}{|c|}{ *: denotes finding is statistically significant, $p \leq 0.05$} \\
\hline \multicolumn{7}{|c|}{ Model 1: unadjusted } \\
\hline \multicolumn{7}{|c|}{ Model 2: adjusted for age \pm 30} \\
\hline \multicolumn{7}{|c|}{ Model 3: adjusted for age $\pm 30, \mathrm{BMI} \pm 30$} \\
\hline
\end{tabular}

\section{Discussion And Conclusions}

The main finding of this study was that participants with EPDS scores indicative of depression were at higher risk for GDM. Preliminary bivariate analyses support the evidence base with regards to the connections between older maternal age and higher pre-pregnancy BMI with greater GDM risk. The association between depression and GDM risk was further strengthened after additional modeling adjustments for maternal age and BMI. While less robust than the depression findings, this work also suggests ACEs and social support during pregnancy may influence GDM risk. We encourage future research in this area. 
Based on the extensive literature review conducted, this study appears to be the first prospective examination of the impact of multiple psychosocial factors, including maternal depression, ACEs, and social support, on the development of GDM. Research on health issues is increasingly acknowledging the contribution that psychosocial factors may have on the development of diseases, and it is critical to expand understanding in these areas to better inform preventative interventions.

While continuous EPDS scores did not show significant relationships with GDM, an examination of binary EPDS scores indicative of depression did reveal significant associations across all three models (Table 2). The fully adjusted Model 3 showed women with depression had an almost 3.2-fold increased risk of developing GDM, corroborating research in this area that also controlled for covariates of maternal BMI and age (Bowers et al., 2013; Byrn \& Penckofer, 2015). In contrast, some literature notes a significantly higher risk for GDM in depressed women who were non-obese (Hinkle et al., 2016), or found that the relationship between GDM and depression became nonsignificant after controlling for weight-related variables like pre-pregnancy BMI and gestational weight gain (Morrison et al., 2016). These variations may be due to other population nuances.

Research has shown a likely bidirectional relationship between depression and diabetes, with both physiologic and psychosocial pathways (Byrn \& Penckofer, 2015), which is consistent with the biopsychosocial perspective. Metabolic control of diabetes has been shown to be affected by psychophysiological processes, as well as individual adherence to prescribed treatment regimens, inclusive of both medications and behaviors like diet and physical activity (Peyrot et al., 1999). Stress levels and coping abilities influence metabolic control and these pathways (Peyrot et al., 1999). Depression has been linked to hyperglycemia (Lustman et al., 2000); both hyperglycemia and insulin resistance are associated with GDM. Future research can better elucidate the relationship dynamics.

Given that by definition ACEs occur prior to age 18, these instances of adversity may be thought of as being remote to pregnancy, particularly regarding a study sample with an average age of 28.5 years. However, as our knowledge increases of the potential latent biological and psychosocial ramifications of ACEs (Anda et al., 2006; CDC, 2019; Felitti et al., 1998), their possible influence via behavioral and/or physiological pathways related to pregnancy health warrants examination. While none of the models reached statistical significance, the binary ACE variable may hint at trends in support of this hypothesis (Table 3).

A connection between social support and GDM could also potentially occur directly through physiological processes, or indirectly through behavioral and/or psychosocial factors that impact physiology. There is evidence supporting the hypothesis that higher levels of maternal social support can decrease the risk for GDM through increased physical activity (Harrison et al., 2018; Omidvar et al., 2018; Smith et al., 2005) and/or healthy lifestyle behaviors (Kaiser et al., 2016; Omidvar et al., 2018). However, the current study analyses examining associations between social support and GDM are firmly non-significant (Table 4), perhaps suggesting that maternal social support is not as relevant to the pathophysiology of GDM.

Pearson's correlation assessments showed EPDS scores were highly positively correlated with ACE scores (significant at the 0.01 level, 2-tailed), and were highly negatively correlated with ISEL scores (significant at the 0.01 level, 2-tailed; data not shown). As a participant's score on the EPDS increased, so did their total number of ACE categories, and their tendency to have a lower level of social support. This reinforces the strong interrelationship between these psychosocial variables. This study found significant relationships between depression and GDM, but despite the strong correlations between maternal depression, ACE scores, and ISEL scores, significant relationships between ACEs or social support with GDM were not found in regression analyses. Recent explorations highlight that irrespective of duration or intensity, adversity exposures of both moderate and high levels during any period of childhood heighten the risk for depression later in life (Tracy et al., 2019). Bădescu et al. (2016) report on the connection between increased chronic stress levels contributing to inflammatory pathways that cause insulin resistance. Women with low levels of perceived social support report more depressive symptoms, but research indicates that networks of strong social support provide a protective effect against pregnancy complications (Elsenbruch et al., 2007). While this study finds an association between depression and risk for GDM, a history of ACEs or perceived level of social support may be a step removed from this risk relationship.

\section{Study Limitations}

This study has several limitations to note. The prevalence of GDM was low in the study population, which placed limits on analytical power and the ability to identify associations. A non-random, convenience sample comprised the study participants, and the OGTT used to diagnose GDM was performed as part of routine prenatal care, and was not specifically conducted as part of the study protocol. Additionally, measures of the psychosocial variables were obtained via self-report on the prenatal questionnaires. It is possible that different measurements of these variables would be obtained using clinical diagnosis. The generalizability of results may be limited given that all study participants were English-speaking and were receiving prenatal care.

\section{Study Strengths \& Implications}

Data for this study were collected prospectively, and included a multimodal data collection protocol which included both self-report questionnaires and medical record abstractions. Additionally, a large proportion of participants were low income and of minority race. Further investigation of psychosocial factors in larger, diverse populations is warranted, including examination of the impact of interventions at various time-points throughout the life course.

This study provides important contributions, specifically addressing some of the shortcomings in the knowledge base of the potential implications for psychosocial factors in GDM development. Findings support the literature connecting GDM and maternal depression, and that maternal age and BMI strengthens this association. These findings underscore the importance of considering both psychosocial factors and biological variables in GDM development.

Pantell et al. (2019) examined the value of combining both clinical and social factors in order to more precisely predict risk; the results of regression models showed a graded association: as the number of social and behavioral risk factors increased, so did the risk of developing diabetes. Future research in women's health should build upon these ideas to increase our understanding of the complex interactions between biological, psychological, and social health factors. 
Potential mechanisms for associations between the factors examined in this study could be further explored to determine causal or mediating/ moderating relationships. Additionally, interventions for psychosocial factors found to be associated with the development of GDM may show a greater impact on development and/or control of GDM as compared to current recommendations that focus primarily and/or exclusively on health behaviors like physical activity and diet. Taking a holistic, biopsychosocial approach may prove effective in guiding future research and interventions to address pregnancy complications like GDM.

\section{Declarations}

Ethics approval: This is an observational study using an existing dataset. The Institutional Review Board, Office of Regulatory and Research Compliance, Division for Research, University at Albany, State University of New York, has confirmed that this study qualifies as exempt from review as per Exempt Category 4 as defined in 45 CFR $\S 46.101$ (b). This study was performed in accordance with the ethical standards as laid down in the 1964 Declaration of Helsinki and its later amendments

\section{Consent for publication: Not Applicable}

Conflicts of interest/competing interests: Not Applicable

Availability of data and material: The datasets analyzed during the current study are available from the corresponding author on reasonable request.

Funding: Dr. Appleton was supported by a JPB Environmental Health Fellowship award granted by The JPB Foundation and managed by the Harvard T.H. Chan School of Public Health.

Author's contributions: AAA designed and fielded the AIMS study, and collected the data. MV conceived and designed the current study, performed the analysis, and wrote the manuscript, with significant guidance and critical revision from dissertation committee members CTB, HL, and AAA. All authors read and approved the final manuscript.

Acknowledgements: Not Applicable

\section{References}

American Diabetes Association (ADA). (2016). What is Gestational Diabetes? Diabetes.org. Retrieved from http://www.diabetes.org/diabetesbasics/gestational/what-is-gestational-diabetes.html?referrer=https://www.google.com/

Anda, R. F., Felitti, V. J., Bremner, J. D., Walker, J. D., Whitfield, C., Perry, B. D., ... Giles, W. H. (2006). The enduring effects of abuse and related adverse experiences in childhood: A convergence of evidence from neurobiology and epidemiology. European Archives of Psychiatry and Clinical Neuroscience, 256(3), 174-186. http://doi.org/10.1007/s00406-005-0624-4

Appleton, A.A., Kiley, K., Holdsworth, E.A., \& Schell, L. M. (2019). Social support during pregnancy modifies the association between maternal adverse childhood experiences and infant birth size. Maternal and Child Health Journal, 23(3), 408-415.

Bădescu, S., Tătaru, C., Kobylinska, L., Georgescu, E., Zahiu, D., Zăgrean, A., \& Zăgrean, L. (2016). The association between Diabetes mellitus and Depression . Journal of Medicine and Life, 9(2), 120-125. https://www.ncbi.nlm.nih.gov/pmc/articles/PMC4863499/

Bardenheier, B. H., Elixhauser, A., Imperatore, G., Devlin, H. M., Kuklina, E. V., Geiss, L. S., \& Correa, A. (2013). Variation in prevalence of gestational diabetes mellitus among hospital discharges for obstetric delivery across 23 states in the United States. Diabetes Care, 36(5), 1209-1214. https://doi.org/10.2337/dc120901

Bergink, V., Kooistra, L., Lambregtse-van den Berg, M. P., Wijnen, H., Bunevicius, R., van Baar, A., \& Pop, V. (2011). Validation of the Edinburg depression scale during pregnancy. Journal of Psychosomatic Research, 70, 385-389. doi:10.1016/j.jpsychores.2010.07.008

Bo, S., Menato, G., Lezo, A., Signorile, A., Bardelli, C., De Michieli, F., ..., \& Pagano, G. (2001). Dietary fat and gestational hyperglycaemia. Diabetologia, 44(8), 972-978. https://doi.org/10.1007/s001250100590

Bowers, K., Laughon, S. K., Kim, S., Mumford, S. L., Brite, J., Kiely, M., \& Zhang, C. (2013). The Association between a Medical History of Depression and Gestational Diabetes in a Large Multi-ethnic Cohort in the United States. Paediatric and Perinatal Epidemiology, 27(4), 323-328.

http://doi.org/10.1111/ppe.12057

Byrn, M. \& Penckofer, S. (2015). The relationship between gestational diabetes and antenatal depression. Journal of Obstetric, Gynecologic \& Neonatal Nursing, 44(2), 246-255. https://doi.org/10.1111/1552-6909.12554

Centers for Disease Control and Prevention (CDC). (2019). Adverse Childhood Experiences (ACEs). National Center for Injury Prevention and Control, Division of Violence Prevention. Retrieved from https://www.cdc.gov/violenceprevention/acestudy/index.html

Centers for Disease Control and Prevention (CDC). (2017). Diabetes home. Who's at risk? Retrieved from https://www.cdc.gov/diabetes/basics/riskfactors.html 
Chou, F. H., Avant, K. C., Kuo, S. H., \& Fetzer, S. J. (2008). Relationships between nausea and vomiting, perceived stress, social support, pregnancy planning, and psychosocial adaptation in a sample of mothers: A questionnaire survey. International Journal of Nursing Studies, 45, 1185-

1191. https://doi.org/10.1016/j.ijnurstu.2007.08.004

Chu, S. Y., Callaghan, W. M., Kim, S. Y., Schmid, C. H., Lau, J., England, L. J., \& Dietz, P. M. (2007). Maternal obesity and risk of gestational diabetes mellitus. Diabetes Care, 30(8), 2070-2076. https://doi.org/10.2337/dc06-2559a

Cohen, S., \& Hoberman, H. M. (1983). Positive events and social supports as buffers of life change stress. Journal of Applied Social Psychology, $13(2)$, 99-25. http://repository.cmu.edu/cgi/viewcontent.cgi?article=1264\&context=psychology

Cox, J. L., Chapman, G., Murray, D., \& Jones, P. (1996). Validation of the Edinburgh postnatal depression scale (EPDS) in non-postnatal women. Journal of Affective Disorders, 39(3), 185-189.https://doi.org/10.1016/0165-0327(96)00008-0

Cox, J. L., Holden, J. M., \& Sagovsky, R. (1987). Detection of postnatal depression - Development of the 10-item Edinburgh postnatal depression scale. British Journal of Psychiatry, 150, 782-786. https://illiad-albany-edu.libproxy.albany.edu/illiad/illiad.dll?Action=10\&Form=75\&Value=912120

DeSisto, C. L., Kim, S. Y., \& Sharma, A. J. (2014). Prevalence estimates of gestational diabetes mellitus in the United States, Pregnancy Risk Assessment Monitoring System (PRAMS), 2007-2010. Preventing Chronic Disease, 11:130415. DOl:http://dx.doi.org/10.5888/pcd11.130415

Elsenbruch, S., Benson, S., Rücke, M., Rose, M., Dudenhausen, J., Pincus-Knackstedt, M. K., ..., \& Arck, P. C. (2007). Social support during pregnancy: effects on maternal depressive symptoms, smoking and pregnancy outcome. Human Reproduction, 22(3), 869-877. https://doi.org/10.1093/humrep/del432

Engel, G. L. (1977). The need for a new medical model: A challenge for biomedicine. Science, 196(4286), 129-136.

http://www.jstor.org.libproxy.albany.edu/stable/1743658

Felitti, V., \& Anda, R. (2010). The relationship of adverse childhood experiences to adult medical disease, psychiatric disorders and sexual behavior: Implications for healthcare. In R. Lanius, E. Vermetten, \& C. Pain (Eds.), The Impact of Early Life Trauma on Health and Disease: The Hidden Epidemic (pp. 7787). Cambridge: Cambridge University Press. doi:10.1017/СВ09780511777042.010

Felitti, V. J., Anda, R. F., Nordenberg, D., Williamson, D. F., Spitz, A. M., Edwards, V., ... \& Marks, J. S. (1998). Relationship of childhood abuse and household dysfunction to many of the leading causes of death in adults. The Adverse Childhood Experiences (ACE) Study. American Journal of Preventative Medicine, 14(4), 245-258. DOI: http://dx.doi.org/10.1016/S0749-3797(98)00017-8

Harrison, A. L., Taylor, N. F., Shields, N., \& Frawley, H. (2018). Attitudes, barriers and enablers to physical activity in pregnancy women: a systematic review. Journal of Physiotherapy, 64(1), 24-32. https://doi.org/10.1016/j.jphys.2017.11.012

Hinkle, S. N., Buck Louis, G. M., Rawal, S., Zhu, Y., Albert, P. S., \& Zhang, C. (2016). A longitudinal study of depression and gestational diabetes in pregnancy and the postpartum period. Diabetologia, 59 (12), 2594-2602. https://doi.org/10.1007/s00125-016-4086-1

Horsch, A., Kang, J. S., Vial, Y., Ehlert, U., Borghini, A., Marques-Vidal, P., ..., \& Puder, J. J. (2016). Stress exposure and psychological stress responses are related to glucose concentrations during pregnancy. British Journal of Health Psychology, 21, 712-729. doi:10.1111/bjhp.12197

Hosler, A. S., Nayak, S. G. \& Radigan, A. M. (2011). Stressful events, smoking exposure and other maternal risk factors associated with gestational diabetes mellitus. Paediatric and Perinatal Epidemiology, 25, 566-574. doi:10.1111/j.1365-3016.2011.01221.x

Kaiser, B., Jeannot, E., \& Razurel, C. (2016). Determinants of health behaviors after gestational diabetes mellitus: A prospective cohort study in Geneva. Journal of Midwifery and Women's Health, 61, 571-577. DOI: 10.1111/jmwh.12486

Lustman, P. J., Anderson, R. J., Freedland, K. E., de Groot, M., Carney, R. M., \& Clouse, R. E. (2000). Depression and poor glycemic control: a meta-analytic review of the literature. Diabetes Care, 23(7), 934-942. https://doi.org/10.2337/diacare.23.7.934

Mason, S. M., Tobias, D. K., Clark, C. J., Zhang, C., Hu, F. B., \& Rich-Edwards, J. W. (2016). Abuse in childhood or adolescence and gestational diabetes. American Journal of Preventive Medicine, 50(4), 436-444. DOI: https://doi.org/10.1016/j.amepre.2015.08.033

Mizuno, S., Nishigori, H., Sugiyama, T., Takahashi, F., Noriyuki, I., Watanabe, Z., ... \& Yaegashi, N. (2016). Association between social capital and the prevalence of gestational diabetes mellitus: An interim report of the Japan environment and children's study. Diabetes Research and Clinical Practice, $120,132-141$. https://doi.org/10.1016/j.diabres.2016.07.020

Morrison, C., McCook, J. G., \& Bailey, B. A. (2016). First trimester depression scores predict development of gestational diabetes mellitus in pregnancy rural Appalachian women. Journal of Psychosomatic Obstetrics \& Gynecology, 37(1), 21-25. DOI: 10.3109/0167482X.2015.1106473

Omidvar, S., Faramarzi, M., Hajian-Tilak, K., \& Amiri, F. N. (2018). Associations of psychosocial factors with pregnancy healthy life styles. PLoS ONE 13(1), e0191723. https://doi.org/10.1371/journal.pone.0191723

Pantell, M.S., Prather, A.A., Downing, J.M., Gordon, N.P., \& Adler, N.E. (2019). Association of social and behavioral risk factors with earlier onset of adult hypertension and diabetes. Journal of the American Medical Association Network Open, 2(5), e193933. doi:10.1001/jamanetworkopen.2019.3933 
Peyrot, M., McMurry, J. F. Jr., \& Kruger, D. F. (1999). A biopsychosocial model of glycemic control in diabetes: stress, coping and regimen adherence. Journal of Health and Social Behavior, 40(2), 141-158. http://content.ebscohost.com/ContentServer.asp?

$\mathrm{T}=\mathrm{P} \& \mathrm{P}=\mathrm{AN} \& \mathrm{~K}=10467761 \& S=\mathrm{R} \& \mathrm{D}=\mathrm{mnh} \&$ EbscoContent=dGJyMNLr40SeqLI4v\%2BbwOLCmr1Cep7BSr664S6\%2BWxWXS\&ContentCustomer=dGJyMPGqtk\%2E

Sattler, M. C., Jelsma, J. G. M., Bogaerts, A., Simmons, D., Desoye, G., Corcoy, R., .., \& van Poppel, M. N. M. (2017). Correlates of poor mental health in early pregnancy in obese European women. BMC Pregnancy and Childbirth, 17, 404. https://doi.org/10.1186/s12884-017-1595-y

Sauder, K., Starling, A., Shapiro, A., Kaar, J., Ringham, B., Glueck, D., ... Dabelea, D. (2016). Diet, physical activity and mental health status are associated with dysglycaemia in pregnancy: The Healthy Start Study. Diabetic Medicine: A Journal of the British Diabetic Association, 33(5), 663-667.

http://doi.org/10.1111/dme.13093

Smith, B. J., Cheung, N. W., Bauman, A. E., Zehle, K., \& McLean, M. (2005). Postpartum physical activity and related psychosocial factors among women with recent gestational diabetes mellitus. Diabetes Care, 28(11), 2650-2654. Dol: 10.2337/diacare.28.11.2650

Tracy, M., Salo, M., Slopen, N., Udo, T., \& Appleton, A. (2019). Trajectories of childhood adversity and the risk of depression in young adulthood: Results from the Avon Longitudinal Study of Parents and Children. Depression and Anxiety, 1-11. DOI: 10.1002/da.22887 